\title{
De novo myoepithelial carcinoma with multiple metastases arising from a submandibular salivary gland: A case report
}

\author{
KARINA CECÍLIA PANELLI SANTOS ${ }^{1}$, HIDENOBU MATSUZAKI ${ }^{2}$, TERUHISA UNETSUBO ${ }^{1}$, \\ SHIMO TSUYOSHI ${ }^{3}$, HITOSHI NAGATSUKA ${ }^{4}$ and JUN-ICHI ASAUMI ${ }^{1}$
}

${ }^{1}$ Department of Oral and Maxillofacial Radiology, Field of Tumor Biology, Okayama University Graduate School of Medicine, Dentistry and Pharmaceutical Sciences; ${ }^{2}$ Department of Oral Diagnosis and Dentomaxillofacial Radiology, Okayama University Hospital; Departments of ${ }^{3}$ Oral and Maxillofacial Surgery and Biopathology and

${ }^{4}$ Oral Pathology and Medicine, Okayama University Graduate School of Medicine,

Dentistry and Pharmaceutical Sciences, Kita-ku, Okayama 700-8558, Japan

Received December 16, 2014; Accepted December 18, 2016

DOI: $10.3892 / 01.2017 .5783$

\begin{abstract}
Salivary gland carcinomas are rare tumors, representing $\sim 0.5 \%$ of all malignancies. Myoepithelioma is also uncommon, representing $\sim 1 \%$ of all salivary gland tumors. Myoepithelial carcinoma (MC) is even rarer, representing 0.2 to $0.6 \%$ of all salivary gland tumors. We herein report a case of MC with multiple metastases arising from a submandibular gland in a 71-year-old male patient and present the associated imaging findings. The patient was considered to have a de novo type of myoepithelial carcinoma, which is reportedly associated with higher malignancy than the transformation type of the disease (i.e., a malignant change from pleomorphic adenoma or myoepithelioma). This was reflected in the multiple lung and bone metastases sites and strong positivity for p53 and Ki-67.
\end{abstract}

\section{Introduction}

Salivary gland carcinomas are rare tumors, representing $\sim 0.5 \%$ of all malignancies and $<5 \%$ of all head and neck cancers (1). The epidemiology of salivary gland tumors is not well documented. According to the World Health Organization (WHO), however, malignant tumors comprise 15 to $32 \%$ of parotid salivary gland tumors, 41 to $45 \%$ of submandibular salivary gland tumors, 70 to $90 \%$ of sublingual salivary gland tumors, and $50 \%$ of minor salivary gland tumors (2).

Correspondence to: Dr Karina Cecília Panelli Santos, Department of Oral and Maxillofacial Radiology, Field of Tumor Biology, Okayama University Graduate School of Medicine, Dentistry and Pharmaceutical Sciences, 5-1 Shikata-Cho, 2-Chome, Kita-ku, Okayama 700-8558, Japan

E-mail:kapanelli@hotmail.com

Key words: myoepithelial carcinoma, submandibular glands, dynamic magnetic resonance imaging
Myoepithelioma is a rare tumor, representing $\sim 1 \%$ of all salivary gland tumors. Myoepithelial carcinoma (MC) is even rarer, representing $\sim 0.2$ to $0.6 \%$ of all salivary gland tumors. MC most commonly develops in the parotid gland, followed by the minor salivary glands and submandibular salivary glands. MC is locally aggressive and characterized by infiltrative growth and the potential for metastasis (2). It was first described in 1975 by Stromeyer et al (3). Males and females are affected equally, with a wide age range of 14 to 86 years (2). The current diagnostic criterion for $\mathrm{MC}$ is the detection of exclusive myoepithelial differentiation by immunohistochemical analysis. However, three-dimensional images including those obtained by magnetic resonance imaging (MRI), computed tomography (CT) and positron emission tomography/CT (PET/CT) are useful for determining the tumor's extension and degree of invasion as well as identifying possible metastases and recurrence $(4,5)$. Additionally, dynamic contrast-enhanced MRI (DCE-MRI) and diffusion-weighted imaging (DWI) have been proposed as efficient tools for the diagnosis of salivary gland tumors (6-8).

We herein report a case involving a patient with a submandibular salivary gland MC with multiple metastases.

\section{Case report}

In March 2013, a 71-year-old male was referred to Okayama University Hospital, Japan, for diagnosis and treatment of a submandibular mass. The patient had noted a mass that was somewhat larger than his submandibular lymph nodes located in his left mandibular angle region in February 2012. Patient anamnesis revealed critical information with regard to his medical history, including hypertension, hyperlipidemia and a cerebral infarction with resultant stent placement in the left carotid artery in June 2008. The patient underwent medical treatment with amlodipine besylate, clopidogrel sulfate, loxoprofen and rebamipide. He was addicted to alcohol and tobacco. Intraoral examination revealed no significant findings, but extraoral examination demonstrated two masses measuring $32 \times 30 \mathrm{~mm}$ and $30 \times 30 \mathrm{~mm}$ in the left 


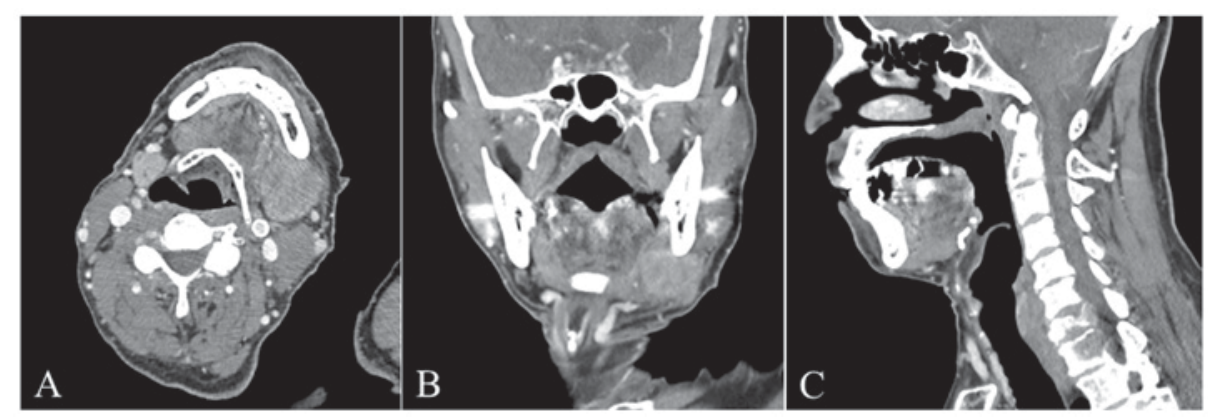

Figure 1. Contrast-enhanced computed tomography images (soft tissue window). (A and B) Axial and coronal images revealed a mass with slight enhancement in the left submandibular salivary gland. (C) Sagittal image revealed bone metastases in the C7 and T1 vertebrae with slight expansion to the vertebral canal.
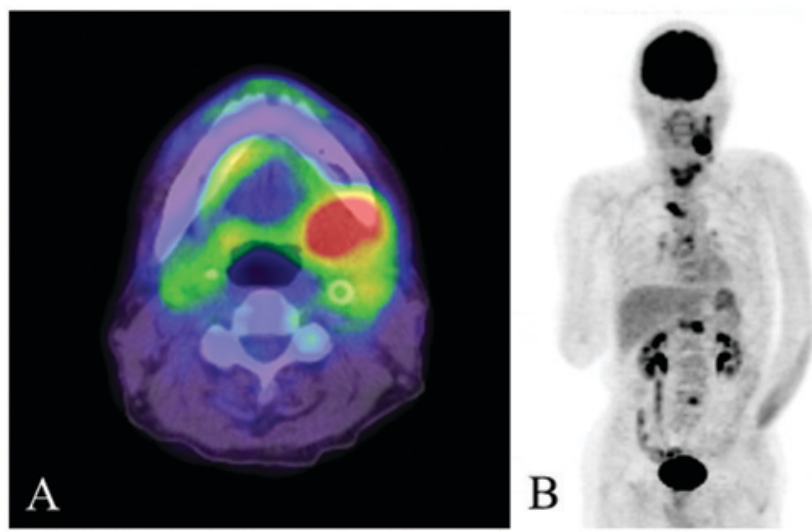

Figure 2. (A) Axial 18F-fluorodeoxyglucose (FDG) positron emission tomography/computed tomography revealed FDG accumulation in the lesion of the left submandibular gland. (B) There were multiple foci of FDG uptake in the lymph nodes and vertebrae of the neck.

submandibular area. The two masses exhibited tenderness, induration and mobility.

The patient underwent imaging examinations involving CT, 18F-fluorodeoxyglucose (FDG) PET/CT and MRI with a dynamic protocol. Contrast-enhanced helical CT images revealed a $10 \times 3 \times 3 \mathrm{~cm}$ tumor of the left submandibular salivary gland and bone metastases in the $\mathrm{C} 7$ and $\mathrm{T} 1$ vertebrae with slight expansion to the vertebral canal (Fig. 1A-C). FDG-PET/CT images demonstrated FDG uptake in the left submandibular salivary gland and left cervical lymph nodes. Multiple uptake points were also observed in the spine and lungs, confirming the presence of metastasis (Fig. 2A and B).

MRI revealed an ill-defined tumor of the left submandibular gland. The mass was heterogeneous and hypo- to isointense on T1-weighted (T1W) images, and heterogeneous and hypo- to hyperintense on short TI inversion-recovery images and T2-weighted (T2W) images (Fig. 3A-C). Heterogeneous enhancement of the internal jugular lymph nodes was observed on contrast-enhanced T1W images (Fig. 3D). Vertebral MRI revealed bone metastasis in the C7, T1, T4, T5, T6, T12 and L4 vertebrae with expansion to the dorsal side; spinal canal stenosis was also present. In addition, vertebral compression fractures were observed in the $\mathrm{C} 7$ and $\mathrm{T} 12$ vertebrae (Fig. 4A and B).

DCE-MRI was performed with 14 consecutive scans at $1 \mathrm{sec}$ intervals followed by delayed images at 500 and $850 \mathrm{sec}$.

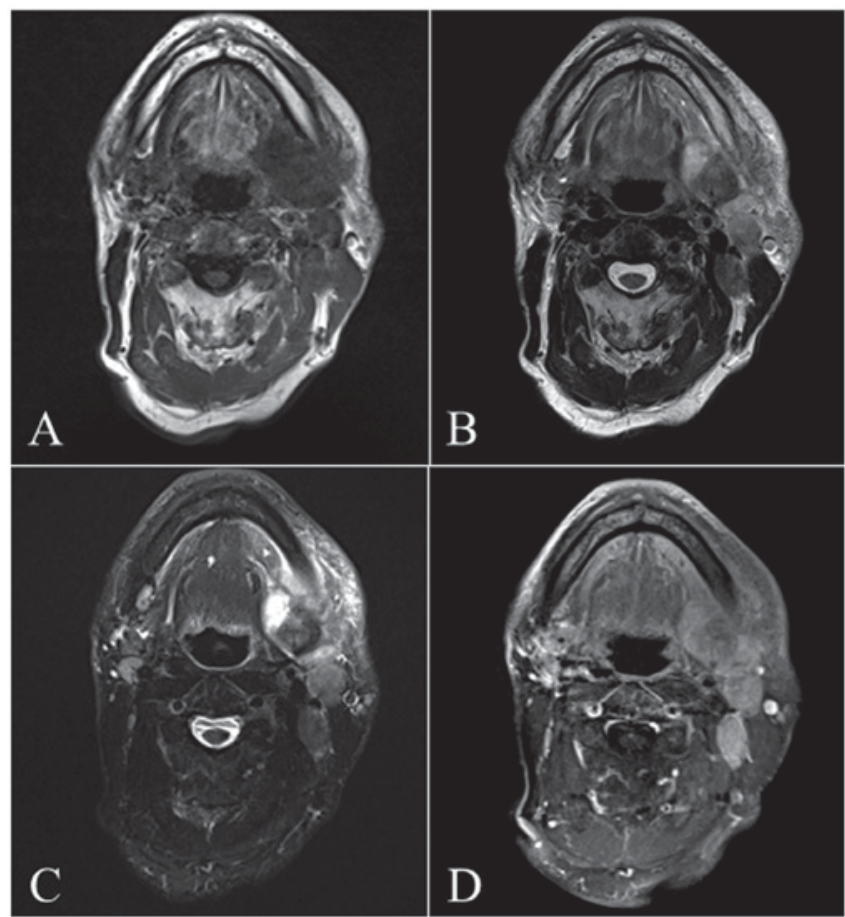

Figure 3. Magnetic resonance imaging of the head and neck. (A-C) A mass was present in the left submandibular gland and exhibited (A) heterogeneous hypo- to isointensity on T1-weighted images and hypo- to hyperintensity on (B) T2-weighted images and (C) short TI inversion-recovery images. (D) The mass exhibited heterogeneous enhancement on contrast-enhanced T1-weighted images.

After determining the region of interest, which was set in the area of greatest enhancement, the time signal intensity curve was obtained. The signal intensity of the curve rapidly increased at 30 to $45 \mathrm{sec}$, then slightly increased until $850 \mathrm{sec}$ (Fig. 5). DWI was performed for the left submandibular salivary gland tumor. The mean apparent diffusion coefficient (ADC) was $1.35 \times 10^{-3} \mathrm{~mm}^{2} / \mathrm{sec}$ (minimum, $1.12 \times 10^{-3} \mathrm{~mm}^{2} / \mathrm{sec}$; maximum, $1.69 \times 10^{-3} \mathrm{~mm}^{2} / \mathrm{sec}$ ).

Fine needle aspiration and incisional biopsy of the submandibular tumor were performed under local anesthesia for microscopic evaluation. Cytological examination revealed a class III alteration. Histopathological examination revealed tumor cells organized in nests of various sizes. The cells contained large and fine chromatin. The tumor had developed within the salivary gland structure. Mitosis was accompanied 


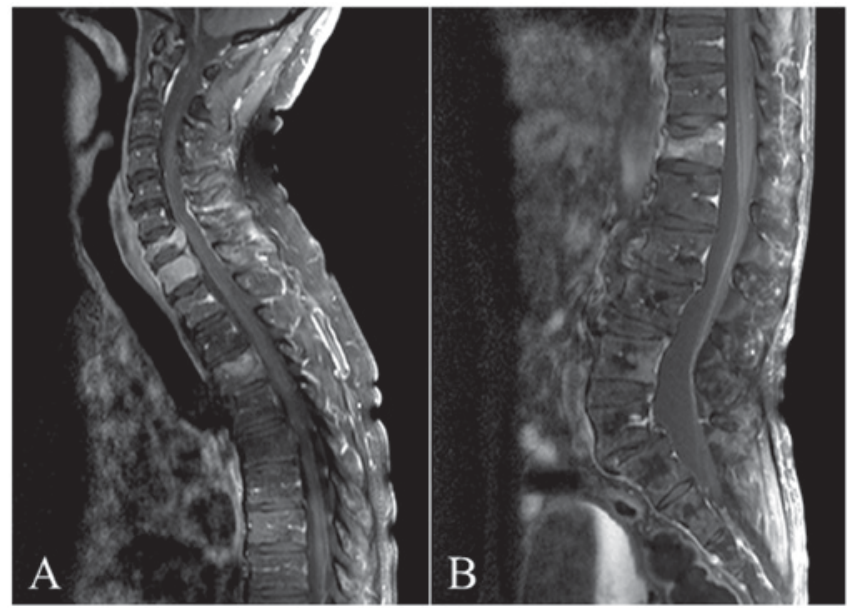

Figure 4. Magnetic resonance images of the vertebrae. (A and B) Multiple bone metastases were present in $\mathrm{C} 7, \mathrm{~T} 1, \mathrm{~T} 4, \mathrm{~T} 5, \mathrm{~T} 6$ and $\mathrm{T} 12$.

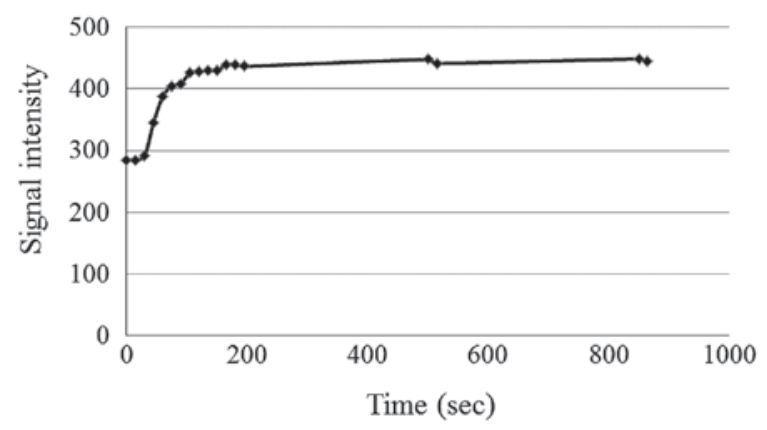

Figure 5. Time signal intensity curve increased for 30 to $40 \mathrm{sec}$ and then gradually increased until $850 \mathrm{sec}$.

by a high degree of cellular atypia. Immunohistochemical evaluation of the tumor revealed strong positivity for $\mathrm{p} 53$ and Ki-67; positivity for cytokeratin (CK) AE1/3, vimentin and epithelial membrane antigen; partial positivity for glial fibrillary acidic protein, S-100, CK7 and CK14; and negativity for smooth muscle actin, carcinoembryonic antigen, calponin, gross cystic disease fluid protein-15, chromogranin and synaptophysin. The patient was determined to have a carcinoma partially differentiated by myoepithelial cells and with high malignancy, as evidenced by the severe vascular invasion. MC was the most reliable diagnosis, even with the nonspecific findings (Fig. 6).

The patient began treatment in April 2013. Treatment included subcutaneous injection of denosumab (120 mg per $1.7 \mathrm{ml}$ ) for the multiple bone metastases and palliative radiotherapy for the spine metastases (a total of $3000 \mathrm{cGy}$ for each affected area: C7-T1 and T12).

\section{Discussion}

Although malignant salivary gland tumors are rare, the WHO recognizes 24 subtypes with different clinical presentations and prognoses $(1,2)$. The incidence of malignant epithelial tumors of the salivary glands ranges from 0.3 to 3.0 per 100,000 individuals annually, confirming its rarity (1). This rarity may be the reason for the lack of knowledge of the
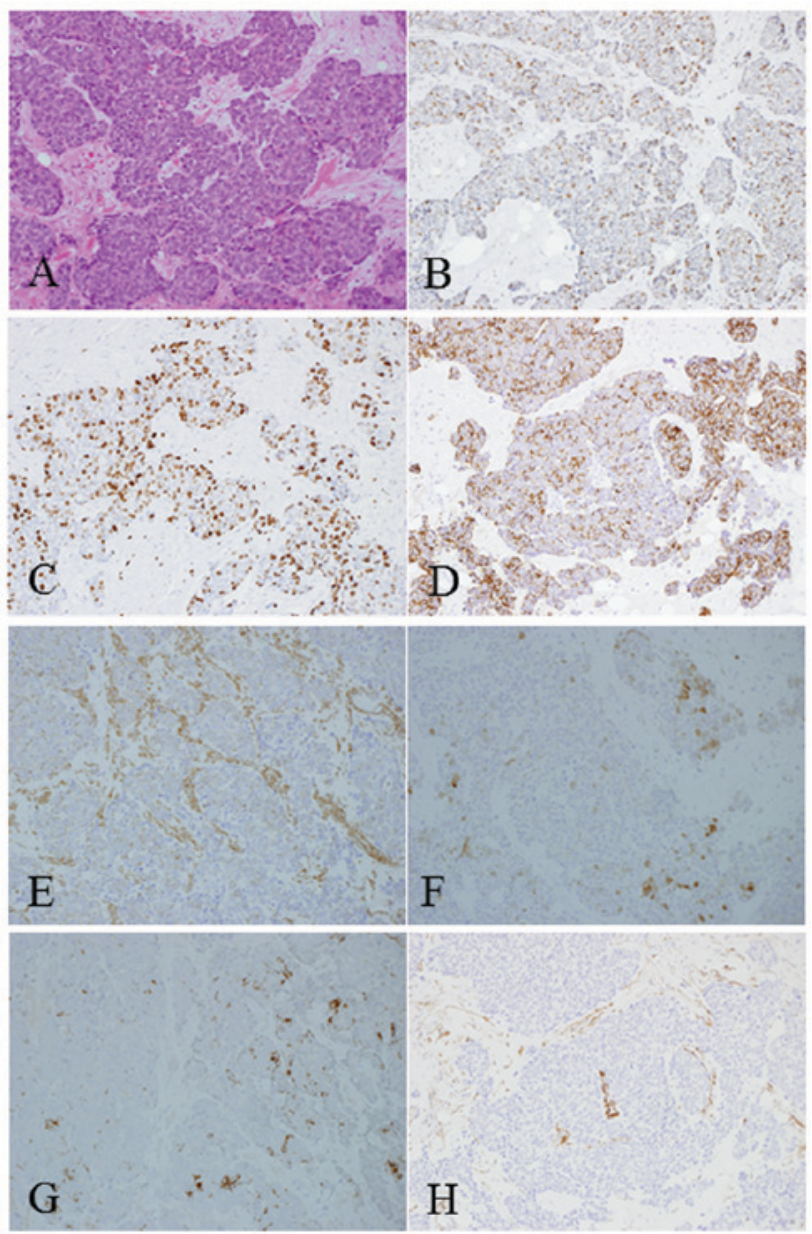

Figure 6. Histological findings. (A) Tumor cells were organized in nests of various sizes and contained large and fine chromatin (hematoxylin-eosin staining; magnification, x200). (B-H) Immunohistochemical examination revealed that the tumor was strongly positive for (B) p53 and (C) Ki-67; positive for (D) cytokeratin AE1/3 and (E) vimentin; partially positive for (F) S-100 and (G) CK14; and negative for $(\mathrm{H})$ smooth muscle actin (magnification, $\mathrm{x} 200$ ).

risk factors for its development $(1,2)$. Ionizing radiation from bombs or medical therapy has been associated with the development of salivary gland cancer, but endogenous hormones and inheritance have only been associated with rare cases of acinic cell carcinoma (1). The rarity and unknown etiology of these tumors were considered in the drafting of the present case report. Notably, this patient had no apparent risk factors for MC other than his previous illnesses and therapies and his current additions to alcohol and tobacco.

$\mathrm{MC}$ is the malignant counterpart of benign myoepithelioma and is composed almost exclusively of tumor cells with myoepithelial differentiation $(2,9)$. There is no gender predilection, and most cases occur in patients from 14 to 77 years of age. The most frequently affected site is the parotid gland, although MC may be identified in all major and minor salivary glands $(2,9)$. The majority of patients present with a painless mass (2). The present patient met all of these criteria with the exception of mass tenderness detected during the extraoral examination.

MC exhibits various histological patterns, including trabecular, reticular and solid $(2,8)$. This variation reflects 
that observed in myoepithelioma, the benign counterpart of MC $(2,8)$. Other typical features are the presence of a multinodular architecture with hypercellular peripheral rims $(9,10)$ and high mitotic activity with considerable variation and marked pleomorphism (2). The MC in the present case was characterized by a multinodular architecture with a solid pattern, a high degree of mitosis and a high atypia grade as indicated by $\mathrm{Ki}-67$ labeling. These findings are in agreement with those of previous studies (9-12).

Histological evaluation should be supported by imaging examination for a complete pretreatment diagnosis. The role of imaging of salivary gland tumors is precise determination of the tumor location, detection of malignant features, assessment of local extension and invasion and detection of nodal metastases and systemic involvement $(1,13)$. MRI and CT are useful to delineate the complete extension of the tumor and identify regional lymphadenopathy. CT is a reasonable alternative in cases of bone infiltration. Combined PET/CT has improved diagnostic accuracy for local tumor extension, nodal involvement and distant metastases, particularly in high-grade malignancies $(1,5,14)$. Unfortunately, the application of PET/CT for salivary gland tumors is still rare, possibly due to its relatively low 18F-FDG uptake, which may be easily obscured by the normal physiological uptake of the salivary glands $(5,14)$. As demonstrated in the present case, CT and PET/CT examination played a significant role in the diagnosis of metastasis, including the metastases to the spine and lung.

MRI is superior with respect to soft tissue differentiation and is useful for detecting deep tissue extension and perineural spread using high-resolution techniques. It also detects signal changes and extracapsular spread in the regional lymph nodes (13). The MRI features of salivary gland tumors include an ill-defined infiltrative margin and hypointensity on T1W and T2W images, which reflect the high malignancy of the tumor $(1,13)$. The lesions typically exhibit heterogeneous enhancement following contrast enhancement (13). The findings described in the present case report are in agreement with the literature; namely hypointensity on T1W and T2W images with heterogeneous enhancement following contrast administration.

DCE-MRI in the oral and maxillofacial areas is reported to be a useful tool with which to differentiate malignant from benign tumors (5,7). Furthermore, Yabuuchi et al (7) suggested that the time-signal intensity curve and appearance of the tumor margin have prognostic value in differentiating among benign tumors, malignant tumors and inflammatory lesions. Malignant tumors generally demonstrate a gradual enhancement-attenuation pattern characterized by early enhancement $(6,7,15)$. In general, the contrast peak of malignant tumors is commonly reached within $120 \mathrm{sec}(6,7)$. In the present case, the lesion demonstrated rapid and gradual enhancement and a contrast peak before $120 \mathrm{sec}$ on DCE-MRI; these features are compatible with malignancy.

Like DCE-MRI, DWI has been used to diagnose malignant tumors in the head and neck region, particularly by using the ADC for the differential diagnosis (8,16-18). The ADC is an essential tool with which to distinguish malignant from benign tumors as it is commonly lower in malignant than benign lesions $(8,16-18)$. The cutoff point is controversial: Wang et al (16) reported an ADC of $1.22 \times 10^{-3} \mathrm{~mm}^{2} / \mathrm{sec}$ using a 1.5 Tesla (T) device, Eida et al (18) reported an ADC of $1.8 \times 10^{-3} \mathrm{~mm}^{2} / \mathrm{sec}$ using a $1.5 \mathrm{~T}$ device, and Srinivasan et al (8) reported an ADC of $1.3 \times 10^{-3} \mathrm{~mm}^{2} / \mathrm{sec}$ using a $3.0 \mathrm{~T}$ device. In the present case, we obtained a mean ADC of $1.35 \times 10^{-3} \mathrm{~mm}^{2} / \mathrm{sec}$. Although this is higher than the values reported by Wang et al (16) and Srinivasan et al (8), it is still extremely close to their results and much lower than that reported by Eida et al (18). After considering the cutoff point, the lesion was classified as malignant.

The optimal treatment for salivary gland tumors should be established following imaging and histological evaluations. Complete surgical excision with clear margins is a well-established treatment for malignant tumors. Considering the tendency for these tumors to recur and metastasize, radiotherapy and chemotherapy are indicated $(1,18)$. However, only supportive treatment in conjunction with palliative radiotherapy was performed in this case.

As shown in our patient's anamnesis, he had undergone stent placement in the left carotid artery in June 2008 to treat a cerebral infarction. Neck CT in May 2008 had revealed no apparent abnormal findings. Therefore, this patient was considered to have a de novo $\mathrm{MC}$. $\mathrm{MC}$ is divided into two types: malignant change from pleomorphic adenoma or myoepithelioma into $\mathrm{MC}$, and de novo $\mathrm{MC}$, which represents 30 to $40 \%$ of all MCs. The de novo type is reportedly associated with higher malignancy than the transformation type (16). The present case revealed strong positivity for p53 and Ki-67, which potentially reflected multiple lung and bone metastases.

In conclusion, a rare case of MC within a submandibular salivary gland has been presented. Histological and imaging evaluations were performed, including DCE-MRI and DWI, which appear to be extremely useful tools in the diagnosis of salivary gland tumors.

\section{References}

1. Ettl T, Schwarz-Furlan S, Gosau M and Reichert TE: Salivary gland carcinomas. Oral Maxillofac Surg 16: 267-283, 2012.

2. Barnes L, Eveson JW, Reichart P and Sidransky D (eds): World Health Organization Classification of Tumours. Pathology and Genetics of Head and Neck Tumours. Vol 9. IARC Press, Lyon, 2005.

3. Stromeyer FW, Haggitt RC, Nelson JF and Hardman JM: Myoepithelioma of minor salivary gland origin. Light and electron microscopical study. Arch Pathol 99: 242-245, 1975.

4. Kane SV and Bagwan IN: Myoepithelial carcinoma of the salivary glands: a clinicopathologic study of 51 cases in a tertiary cancer center. Arch Otolaryngol Head Neck Surg 136: 702-712, 2010.

5. Kim MJ, Kim JS, Roh JL, Lee JH, Cho KJ, Choi SH, Nam SY and Kim SY: Utility of 18F-FDG PET/CT for detecting neck metastasis in patients with salivary gland carcinomas: preoperative planning for necessity and extent of neck dissection. Ann Surg Oncol 20: 899-905, 2013.

6. Hisatomi M, Asaumi J, Yanagi Y, Unetsubo T, Maki Y, Murakami J, Matsuzaki H, Honda Y and Konouchi H: Diagnostic value of dynamic contrast-enhanced MRI in the salivary gland tumors. Oral Oncol 43: 940-947, 2007.

7. Yabuuchi H, Fukuya T, Tajima T, Hachitanda Y, Tomita K and Koga M: Salivary gland tumors: diagnostic value of gadolinium-enhanced dynamic MR imaging with histopathologic correlation. Radiology 226: 345-354, 2003.

8. Srinivasan A, Dvorak R, Perni K, Rohrer S and Mukherji SK: Differentiation of benign and malignant pathology in the head and neck using 3T apparent diffusion coefficient values: early experience. AJNR Am J Neuroradiol 29: 40-44, 2008.

9. Thayumanavan B and Vani NV: Myoepithelial carcinoma of palate: case report. Indian J Pathol Microbiol 57: 85-88, 2014. 
10. Savera AT, Sloman A, Huvos AG and Klimstra DS: Myoepithelial carcinoma of the salivary glands: a clinicopathologic study of 25 patients. Am J Surg Pathol 24: 761-774, 2000.

11. Ren J, Liu Z, Liu X, Li Y, Zhang X, Li Z, Yang Y, Chen Y and Jiang S: Primary myoepithelial carcinoma of palate. World J Surg Oncol 9: 104, 2011.

12. Alós L, Cardesa A, Bombí JA, Mallofré C, Cuchi A and Traserra J: Myoepithelial tumors of salivary glands: a clinicopathologic, immunohistochemical, ultrastructural, and flow cytometric study. Semin Diagn Pathol 13: 138-147, 1996.

13. Lee YY, Wong KT, King AD and Ahuja AT: Imaging of salivary gland tumours. Eur J Radiol 66: 419-436, 2008.

14. Roh JL, Ryu CH, Choi SH, Kim JS, Lee JH, Cho KJ, Nam SY and Kim SY: Clinical utility of 18F-FDG PET for patients with salivary gland malignancies. J Nucl Med 48: 240-246, 2007.
15. Herrera GA: Light microscopic, ultrastructural and immunocytochemical spectrum of malignant lacrimal and salivary gland tumors, including malignant mixed tumors. Pathobiology 58: 312-322, 1990

16. Wang J, Takashima S, Takayama F, Kawakami S, Saito A, Matsushita T, Momose M and Ishiyama T: Head and neck lesions: characterization with diffusion-weighted echo-planar MR imaging. Radiology 220: 621-630, 2001.

17. Maeda M and Maier SE: Usefulness of diffusion-weighted imaging and the apparent diffusion coefficient in the assessment of head and neck tumors. J Neuroradiol 35: 71-78, 2008.

18. Eida S, Sumi M, Sakihama N, Takahashi H and Nakamura T: Apparent diffusion coefficient mapping of salivary gland tumors: prediction of the benignancy and malignancy. AJNR Am J Neuroradiol 28: 116-121, 2007. 